
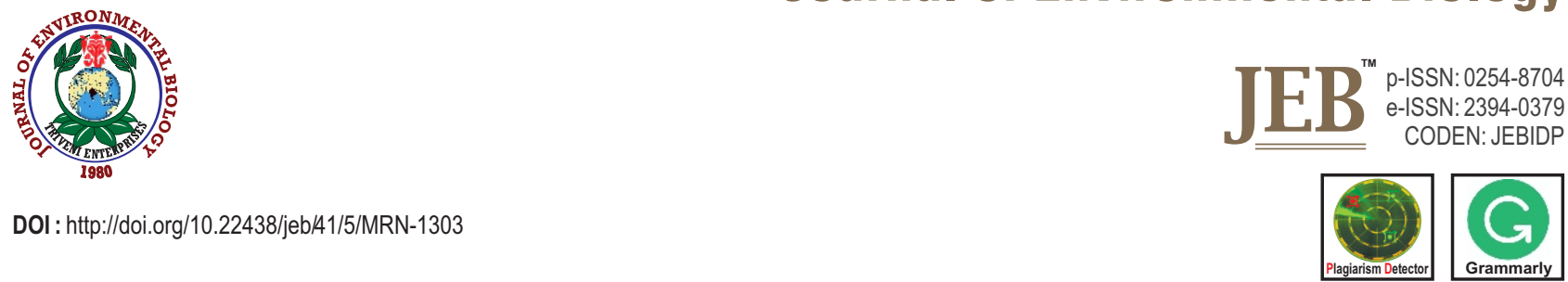

\title{
Biocontrol and growth enhancement potential of Trichoderma spp. against Rhizoctonia solani causing sheath blight disease in rice
}

\author{
S. Chaudhary', S. Sagar', M. Lal', A. Tomar', V. Kumar ${ }^{4}$ and M. Kumar ${ }^{1 *}$ \\ 'Department of Agricultural Biotechnology, College of Agriculture, SVP University of Agriculture and Technology, Meerut-250 110, India \\ 2Department of Recombination Techniques, College of Biotechnology, SVP University of Agriculture and Technology, Meerut-250 110, India \\ ${ }^{3}$ Division of Plant Protection, ICAR-Central Potato Research Institute, Regional Station, Meerut-250 110, India \\ ${ }^{4}$ ICAR-National Institute of Biotic Stress Management, Raipur - 493 225, India
}

*Corresponding Author Email : mukeshbt06@gmail.com

\section{Abstract}

Aim: The present study aimed to investigate the biocontrol and growth enhancement potentials of Trichoderma spp., against $R$. solani.

Methodology: A total of 31 Trichoderma spp. were examined for their antagonistic potentials against $R$. solani in dual culture, effect of volatile and nonvolatile metabolites on the growth inhibition of test pathogen under in-vitro condition. The efficacy of Trichoderma spp. were further evaluated for controlling sheath blight and promoting rice growth under in-vivo conditions with different mode of applications.

Results: The results of in-vitro confrontation assay revealed that among the different Trichoderma spp. tested, T. harzianum (SVPRT-THLi06) and $T$. atroviride (SVPP-4) exhibited excellent biocontrol efficacy with $90.9 \%$ and $72.0 \%$ mycelial growth inhibition, respectively. The culture filtrate of T. harzianum (SVPRT- THLi6) at 50\% was highly effective in reducing $R$. solani mycelial growth up to $95.9 \%$ and in case of volatile metabolites, maximum inhibition (72.5\%) in mycelial growth of $R$. solani was recorded with T. harzianum isolate SVPP-8. A combined mode of application (soil treatment+ root dipping+ foliar spray) with T. harzianum (SVPRT-THLi06) was found most effective under greenhouse condition, which showed the least disease severity ( $12.4 \%$ vs control $49.6 \%$ ) and disease incidence ( $27.1 \%$ vs control $96.4 \%$ ).

Interpretation: The present study revealed that T. harzianum (SVPRTTHLi06) has potential to control sheath blight disease as well as improve growth of rice crop. Therefore, in future it may be used as bio-based formulation for plant growth enhancement and managing sheath blight disease in rice cultivation and further may be applied in organic rice cultivation.

Key words: Necrotrophic fungus, Rhizoctonia solani, Rice, Sheath blight, Trichodermaspp.

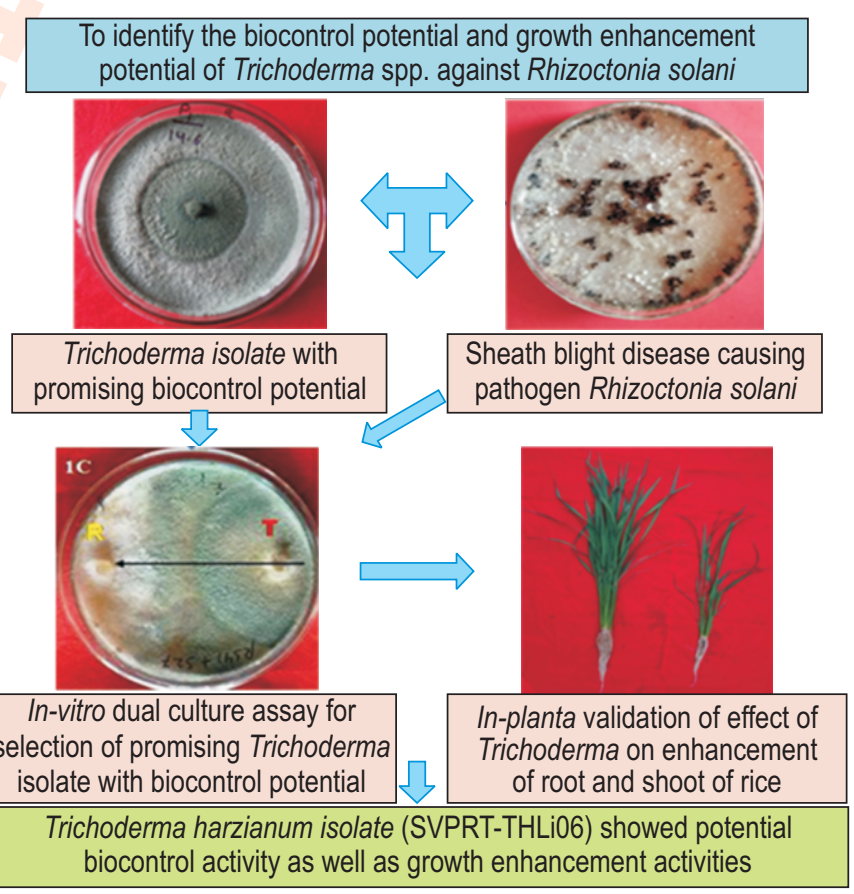

How to cite : Chaudhary, S., S. Sagar, M. Lal, A. Tomar, V. Kumar and M. Kumar: Biocontrol and growth enhancement potential of Trichoderma spp. against Rhizoctonia solani causing sheath blight disease in rice. J. Environ. Biol., 41, 1034-1045 (2020). 


\section{Introduction}

Rice (Oryza sativa L.) is one of the major cereal food crops and form staple diet of about half of the world's population. Globally, the production of rice is estimated 769.6 million tons and rice cultivation area is estimated to be around 167.2 million ha (Chaudhary et al., 2019). India would require about $156 \mathrm{mt}$ of rice by the year 2030 at an annual increment of 3 million tons in the current rice production (Dass et al., 2016, 2017), which should come from yield enhancements as there almost no scope for increasing rice area. However, rice crop is subjected to more than forty diseases, which are one of the biotic factors affecting rice production and productivity in the world including India. Sheath blight caused by soil borne necrotrophic fungal pathogen Rhizoctonia solani Kühn [teleomorph: Thanatephorus cucumeris (A.B. Frank)], is an economically important disease in rice. It can reduce yield up to $25-50 \%$ in Philippines, $20-50 \%$ in Japan and $5.2-50 \%$ in India (Kumar et al., 2013; Richa et al., 2016). Introduction of new, susceptible, high-yielding compact semidwarf varieties, excessive use of nitrogen fertilizers and changes in agricultural practices associated with these varieties contribute to increase in sheath blight disease incidence and severity in ricegrowing areas in the world (Groth and Bond, 2007). Furthermore, changes in climatic conditions also favour the spread of disease. $R$. solani survives in soil within diseased plant debris as mycelium or sclerotia during unfavourable environmental conditions for several years, which serve as source of primary inoculum. Control of rice sheath blight pathogen is difficult due to high genetic variability, extremely broad host range and its ability to survive in soil for a long time under various environmental conditions (Taheri and Tarighi, 2011). So far, absolute resistance to $R$. solani is not available in any of the rice germplasms, although partial genetic resistance has been reported (Yadav et al., 2015; Laha et al., 2017). Owing to lack of desired level of host resistance, the disease is controlled by extreme use of synthetic fungicides, which have negative effect on the soil microflora, pollute the atmosphere, and are environmentally hazardous. Moreover, it has been reported that some effective fungicides are highly phytotoxic to rice and in low severe condition; these fungicides may reduce yields (Groth et al., 1993). Therefore, biological control through antagonistic microorganisms may be an effective approach to minimize the incidence of sheath blight.

Trichoderma is a fungal genus, that includes anamorphic fungi, isolated primarily from soil and decomposing organic matter (Harman et al., 2004). Among the antagonistic microorganisms, the genus Trichoderma is especially known for its biocontrol activities against several phytopathogens, including Rhizoctonia solani (Harman, 2006; Kotasthane et al., 2015; Swain et al., 2018). Trichoderma spp. shows competitive nature for rice plant residue by exhausting the available nutrients for the pathogen, ultimately reducing the survival of sheath blight pathogen (Mostafa and Shahjahan, 1995). In addition,
Trichoderm spp. has been reported to enhance plant growth in maize, wheat, tomatoes, gray mangroves and rice (Raman, 2012; Saravanakumar et al., 2013; Doni et al., 2014; Mahato et al., 2018). In view of the above, the present study aimed to evaluate the potential of different native Trichoderma isolates in controlling rice sheath blight pathogen $R$. solani both in-vitro and in-vivo conditions and to investigate the growth promotion potential of Trichoderma spp. in rice cultivation.

\section{Materials and Methods}

Identification and maintenance of fungal isolates : A total of 31 Trichoderma strains, isolated and characterized in the previous study were used for experiments (Table 1). Pathogenic isolate of Rhizoctonia solani (SVPRS06) was recovered from naturally infected rice plants showing typical symptoms of sheath blight disease in rice fields. To confirm pathogenecity, artificial inoculations were done in greenhouse on rice cv. PB-1 by inserting a 5-mm mycelial disc of $R$. solani (SVPRS06) isolate between the joint of basal leaf sheath and stem above the water line at maximum tillering stage. Re-isolation of $R$. solani was done from artificial inoculated PB-1 rice plants showing distinctive lesions of sheath blight. Anastomosis group (AG) and subgroup of $R$. solani isolate was confirmed by anastomosis reaction with tester AG1-IA isolate (ITCC 7650) procured from ICAR-Indian Agricultural Research Institute (IARI), Pusa Campus, New Delhi, and rDNA sequencing using ITS1 and ITS4 primers (Accession no.- KU215869). R. solani and Trichoderma isolates were grown on potato dextrose agar (PDA) at $28^{\circ} \mathrm{C}$ for 5 days and stored at $4^{\circ} \mathrm{C}$ for further use.

\section{In-vitro plate confrontation assays for biocontrol}

Dual culture assay : In-vitro antifungal efficacy of Trichoderma spp. against $R$. solani was tested by dual culture technique. Mycelial disc ( $\sim 5$ in $\mathrm{mm}$ dia) of $R$. solani and Trichoderma spp. were cut from advancing periphery of 5-day-old PDA culture, and separately positioned opposite to each other $\sim 1.5 \mathrm{~cm}$ away from the edge of $90 \mathrm{~mm}$ Petri plates containing PDA medium. Plates inoculated with $R$. solani (SVPRS06) alone served as control. Each pair was replicated three times and incubated for seven days at $28 \pm 2^{\circ} \mathrm{C}$. The reduction in mycelial growth of $R$. solani was recorded and the percentage of inhibition over control for each treatment was calculated according to Hajieghrari (2010).

The degree of antagonism was scored using the rating system described by Bell et al. (1982) on a scale of 1-5: Class 1: Trichoderma completely overgrew $R$. solani and covered the entire medium surface; Class 2: Trichoderma overgrew at least two third of the medium surface; Class 3: Trichodema and $R$. solani each colonized $50 \%$ of the medium surface and neither organism appeared to dominate the other; Class 4: $R$. solani colonized at least two-third of the medium surface and appeared to withstand encroachment by Trichoderma, and Class 5: $R$. 
Table 1 : Isolate codes and GenBank accession no. for Trichoderma spp. used in the study

\begin{tabular}{lll}
\hline Species & Isolate code & GenBank accession numbers \\
\hline Trichoderma & SVPRT-THLi6, SVPRT-THLi7, SVPRT-THLi8, SVPRT-THLi9, & KC582837, KC582838, KC582839, Kc582840, \\
harzianum & SVPRT-TH1, SVPP-8, SVPP-9, SVPP-10, SVPP-12, SVPP-14, & KX139409, KU215926, KU215920, Ku215921, \\
& SVPP-16, SVPP-18, SVPRT-THL01, SVPRT-THL02, SVPRT- & KU215922, KU215923, KU215924, Ku215925, \\
& THL03, SVPRT-THL04, SVPRT-THL05, SVPRT-THL08, SVPRT- & JX232593, JX232594, JX232595, JX232596, \\
& THL09, SVPP-6 & JX232597, KX139407KX139408, Ku215917 \\
Trichoderma & SVPRT-TL10, SVPRT-LB02, SVPRT-LB06, SVPRT-LB07, & KC582841, JX908722, JX908726, JX908727, \\
longibrachiatum & SVPP-7 & Ku215918 J J \\
Trichoderma asperellum & SVPP-2, SVPP-3, SVPP-5, SVPP-11 & KU215913, KU215915, KU215914, Ku215912 \\
Trichoderma atroviride & SVPP-4 & Ku215916 \\
Trichoderma erinaceum & SVPUAT-TE-01 & Kc121065 \\
\hline
\end{tabular}

solani completely overgrew the entire medium surface. Trichoderma isolates were considered antagonistic to $R$. solani, if the mean score for a given comparison was class $\leq 2$, but not highly antagonistic if the number was class $\geq 3$.

For mycoparasitism test, clean glass slide assay was used. Molten water agar ( $2 \%$ ) was poured and evenly spread over the sterile glass slide to make a thin agar film. The mycelium disks of $R$. solani and Trichoderma isolates were placed on the slide 1 $\mathrm{cm}$ apart from each other and incubate at $28 \pm 2^{\circ} \mathrm{C}$ for 3 days. At the end of the incubation period, the contact/inhibition zone mounted under lectophenol-cotton-blue and observations were made under a light microscope for the presence of coiling structures and wall disintegration in the hyphae of Trichoderma and $R$. solani respectively. The frequencies of coiling or wall disintegration were recorded by observing the coils or areas of disintegration in five different microscopic fields (Sivakumar et al., 2000).

Estimation of volatile metabolites from Trichoderma spp. : The effect of volatile metabolites from Trichoderma spp. against $R$. solani was investigated using paired plate technique (Dennis and Webster, 1971). Trichoderma spp. was centrally inoculated in Petri dish containing PDA medium with $5 \mathrm{~mm}$ mycelial disc taken from 3-day-old actively growing culture. The lids of each Trichoderma inoculated Petri dishes were replaced with bottom of PDA containing Petri dishes inoculated centrally with $5 \mathrm{~mm}$ mycelial disc of $R$. solani and sealed by three layers of parafilm to prevent the loss of volatile metabolites. Petri-plate without Trichoderma spp. served as control. The plates were incubated at $28 \pm 2^{\circ} \mathrm{C}$ with a photoperiod of $12 \mathrm{hr}$. The average diameter of treatments was measured as the time at which the pathogen completely covered the control plate and was further used to calculate the percentage of growth inhibition. Each test was performed in triplicate. The percent inhibition was calculated by the following formula:

$$
\text { Inhibition }(\%)=(\mathrm{C} 1-\mathrm{C} 2) / \mathrm{C} 1 \times 100
$$

where, $\mathrm{C} 1$ means growth of $R$. solani in the presence of
Trichoderma volatiles and C2 means growth of control ( $R$. solani alone).

Estimation of antifungal non-volatile metabolites from Trichoderma spp. : The biocontrol efficacy of non-volatile metabolites from Trichoderma spp. was estimated following the method of Jariwala et al. (1991) with slight modification. Briefly, $1 \mathrm{ml}$ of spore suspension $\left(1 \times 10^{5} \mathrm{cfu} \mathrm{m}^{-1}\right)$ was inoculated in Potato Dextrose Broth (PDB) and incubated at $28 \pm 2^{\circ} \mathrm{C}$ without shaking for 10 days. After incubation, the fungal mycelial mat and spores were removed by filtration through double layer of Whattman filter paper No. 1 and sterilized by passing through a $0.22 \mu \mathrm{m}$ pore size syringe filter (Millipore). The filtrates were used for antifungal activity. Trichoderma culture filtrates were supplemented with molten PDA medium to obtain 5, 10, 25 and $50 \%(\mathrm{v} / \mathrm{v})$ concentration and poured in sterile Petri plates. Mycelial disc of $5 \mathrm{~mm}$ diameter were removed from the edge of 3-day-old actively growing $R$. solani culture and placed at the centre of culture medium and incubated at $28 \pm 2^{\circ} \mathrm{C}$ for $6-7$ days. PDA plates without Trichoderma culture filtrate served as control. The average radial growth of the fungal mycelium was recorded and the percentage inhibition was calculated using the formula described earlier. Each assay was performed in triplicate.

\section{In-vivo evaluation of Trichoderma spp. for their biocontrol and plant growth promotion potential under green house}

Rice seedling and rice plant preparation : Rice seeds (cv. PB1 , high yielding and sheath blight susceptible) from Experimental Research Centre of SVP University of Agriculture and Technology, Meerut, were sown in plastic tray. Pots $(40 \mathrm{~cm}$ dia. $\mathrm{X}$ $30 \mathrm{~cm}$ height) were filled with $10.0 \mathrm{~kg}$ of rice field soil and fertilized by soil application of NPK (100:60:40 kg ha-1). Twenty-one day old rice seedlings were transplanted in each pot (one plant per pot) under wet conditions and watered regularly to keep them always submerged. The pots were arranged in a completely randomized design (CRD) replicated three times for each treatment and kept under green house at 25 to $34^{\circ} \mathrm{C}$ temperature and 85 to $90 \%$ relative humidity. 
Table 2 : Trichoderma species and treatments used for in-vivo biocontrol and plant growth promotion under pot experiment

\begin{tabular}{llll}
\hline S. No. & Treatments & Trichoderma isolate used & Species \\
\hline T1 & Seed + foliar spray & SVPRT-THLi03 & Trichoderma harzianum \\
T2 & Soil + foliar spray & & \\
T3 & Seedling root dip + foliar spray & & \\
T4 & Seedling root dip + soil + foliar spray & SVPRT-THL04 & Trichoderma harzianum \\
T5 & Seed + foliar spray & & \\
T6 & Soil + foliar spray & & \\
T7 & Seedling root dip + foliar spray & SVPRT-THLi06 & Trichoderma harzianum \\
T8 & Seedling root dip + soil + foliar spray & & \\
T9 & Seed + foliar spray & & \\
T10 & Soil + foliar spray & SVPRT-TL10 & Trichoderma longibrachiatum \\
T11 & Seedling root dip + foliar spray & & \\
T12 & Seedling root dip + soil + foliar spray & & \\
T13 & Seed + foliar spray & & Trichoderma atroviride \\
T14 & Soil + foliar spray & & \\
T15 & Seedling root dip + foliar spray & & \\
T16 & Seedling root dip + soil + foliar spray & & \\
T17 & Seed + foliar spray & & \\
T18 & Soil + foliar spray & & \\
T19 & Seedling root dip + foliar spray & Seedling root dip + soil + foliar spray & \\
T20 & & & \\
\hline
\end{tabular}

Pathogen inoculation and application of Trichoderma spp.: Rice plants were inoculated by placing the mycelium disc of $R$. solani (SVPRS06) at the centre of hill above the water level, 30 days after transplanting (Sudhakar et al., 1998). Based on their invitro performance, five potent Trichoderma isolates were selected for antagonistic performance and plant growth promotion activity under in-vivo greenhouse conditions. The selected isolates $T$. harzianum, T. Iongibrachiatum and T. atroviride. Detailed treatment procedure for application of Trichoderma spp. are given in Table 2.

Spore suspensions of Trichoderma isolates were prepared by harvesting the spore from 10-day-old culture grown on PDA by adding sterile water to the plates and scraping the culture with a scalpel. These suspensions were filtered through a triple layer Whattman filter paper No. 1 to separate mycelium from conidia. The concentration of spores was determined by haemocytometer and adjusted to $10^{7}$ conidia $\mathrm{ml}^{-1}$. Tween $20 @$ $0.1 \%$ was supplemented to the suspensions for better adhering of conidia to plant surfaces. Inoculated rice plants were sprayed with spore suspensions of Trichoderma spp. (50 ml per replicate) at two stages: $24 \mathrm{hr}$ after inoculation and 15 days after first spray. Two sprays of Propiconazole 25 EC @ 0.1\% were kept as standard chemical control for comparison. Plants inoculated with $R$. solani only served as control. Seed treatment was done by seed smearing before sowing (15 g talc-based formulation per 1 $\mathrm{kg} \mathrm{seed).} \mathrm{Talc-based} \mathrm{formulation} \mathrm{of} \mathrm{Trichoderma} \mathrm{spp.} \mathrm{was}$ prepared by following the method of Jeyarajan et al. (1994). Soil treatment was done at the time transplanting @ $15 \mathrm{~g} \mathrm{~kg}^{-1}$ of filling and for seedling treatment; 21-day-old rice seedlings were uprooted and dipped in Trichoderma spore suspensions.
Assessment of disease and rice growth components: Disease severity on rice plants was recorded according to (0-4) scale representing severity levels of $0,1,5,15$, and $50 \%$ (Willocquet et al., 2010). Disease severity and disease incidence were recorded on tiller basis. Disease incidence, disease severity and disease reduction in treated and control plants were calculated by the standard formulae (Helfish et al., 2017).

Statistical analysis : Disease incidence and disease severity values were subjected to transformation for statistical analysis. All data were statistically analysed by one-way analysis of variance (ANOVA). The significance of the effect of the treatment was determined using F-test and to determine the significance of the difference between the means of the treatments, least significant difference (LSD) was calculated at $5 \%$ probability level.

\section{Results and Discussion}

Pathogenecity test in greenhouse indicated that $R$. solani (SVPRS06) was a strong virulent pathogen on rice and showed typical symptoms of sheath blight. The BLAST analysis revealed that SVPRS06 showed highest similarity to $R$. solani AG1-IA isolates with reference GenBank accession numbers: KX674520,

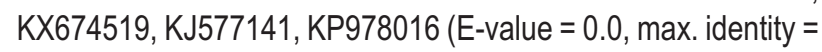
99\%), available at NCBI database.

The data of dual culture assay revealed that all 31 Trichoderma spp. strains tested significantly differed from the control in relation to growth diameter of $R$. solani. However, most $T$. harzianum isolates showed higher growth inhibition of $R$. solani compared to the other isolates. Among the T. harzianum isolates, percent growth inhibition was highest in SVPRT- THLi6 (90.9\%) 
followed by SVPP-8 (81.3\%), SVPRT- THLi8 (77.2\%), SVPRTTHL07 (74.9\%) and SVPRT-THL01 (72.4\%) whereas the minimum growth inhibition (41.9\%) was observed in T. asperellum isolates SVPP-5 at 7 DAI (Table 3). T. harzianum isolate SVPRTTHLi6 showed initial faster growth, overgrew on $R$. solani, destroyed it completely and sporulated after 5 days of inoculation. No inhibition halo zone was observed between SVPRT-THLi6 and $R$. solani, indicated that the antagonistic effect was due to competition for space and nutrient and not on a classic antibiosis or chemical aggressiveness (Fig.1A-1D). T. erinaceum isolate SVPUAT-TE-1 co-culture with $R$. solani also showed faster growth and overgrew on the pathogen (Fig. 1E). A clear zone of interaction between the isolate SVPP-4 and SVPRT-THL01 with pathogen was observed, where both isolates inhibited the growth of $R$. solani after physical contact (Fig. $1 \mathrm{~F}$ and $\mathrm{G}$ ). All $T$. harzianum and $T$. atroviride isolates showed Bell's rating of 1 or 2 , revealing the fact that these isolates had superior antagonistic potential against $R$. solani in-vitro as compared to other isolates of Trichoderma spp. The overgrowth of antagonist is achieved when a fungus exhibits higher growth rate, tolerance against metabolites produced by the pathogens (Mathivanan et al., 2000) and capacity to produce antibiotics. Abo-Elyousr et al. (2014) noted that mycoparasitism in Trichoderma was associated with its ability to produce extra cellular chitinase enzymes. Previous studies have reported inhibitiary action of $T$. harzianum isolates against various phytopathogens like $R$. solani (Yadav et al., 2018), Sclerotinia sclerotiorum (Inbar et al., 1996), Fusarium oxysporum (Prasad etal., 2016) etc., in confrontation assay.
Microscopic analysis showed that hyphae of T. harzianum readily interacted with hyphae of $R$. solani and exhibited successful mycoparasitism. Trichoderma mycelium was distinguished from $R$. solani by hyphal diameter; $T$. harzianum hyphae were thinner as compared to $R$. solani hyphae. $T$. harzianum was capable of degrading $R$. solani hyphae by coiling around the hyphae with apressoria and hook- like structure (Fig. $2 \mathrm{C}, \mathrm{D})$. Apressoria-like structures allowed the hyphae of Trichoderma spp. to adhere to the surface of $R$. solani hyphae. However, some biocontrol agents applied diverse mechanism against $R$. solani as just touched the hyphae without coiling (Fig. $2 \mathrm{E}$ and $\mathrm{F}$ ). As a consequence of mycoparasitic activity, the hyphae of $R$. solani were pierced from the septaum side, causing coagulation of protoplast, resulting in lysis of the host hyphae (Fig. 2G, H). Although, T. atroviride spores accumulated around $R$. solani hyphae, but were not attached to hyphae (Fig. 21). Similar observations were noted against $R$. solani by Khan and Sinha (2007) and Asad et al. (2014). Elad et al. (1983) reported $R$. solani hyphal coiling and penetration by Trichoderma harzianum and T. hamatum and appressorium-like structures and hooks. However, T. atroviride (SVPP-4) did not coil around the hyphae of $R$. solani, hence, the main mechanism of biocontrol for this strain is antibiosis or production of hydrohytic enzymes (Harman et al., 2004).

The culture filtrates of Trichoderma spp. inhibited mycelium growth of $R$. solani and inhibition percentage increased proportionally with the filtrate concentration (Fig. 3). T. harzianum
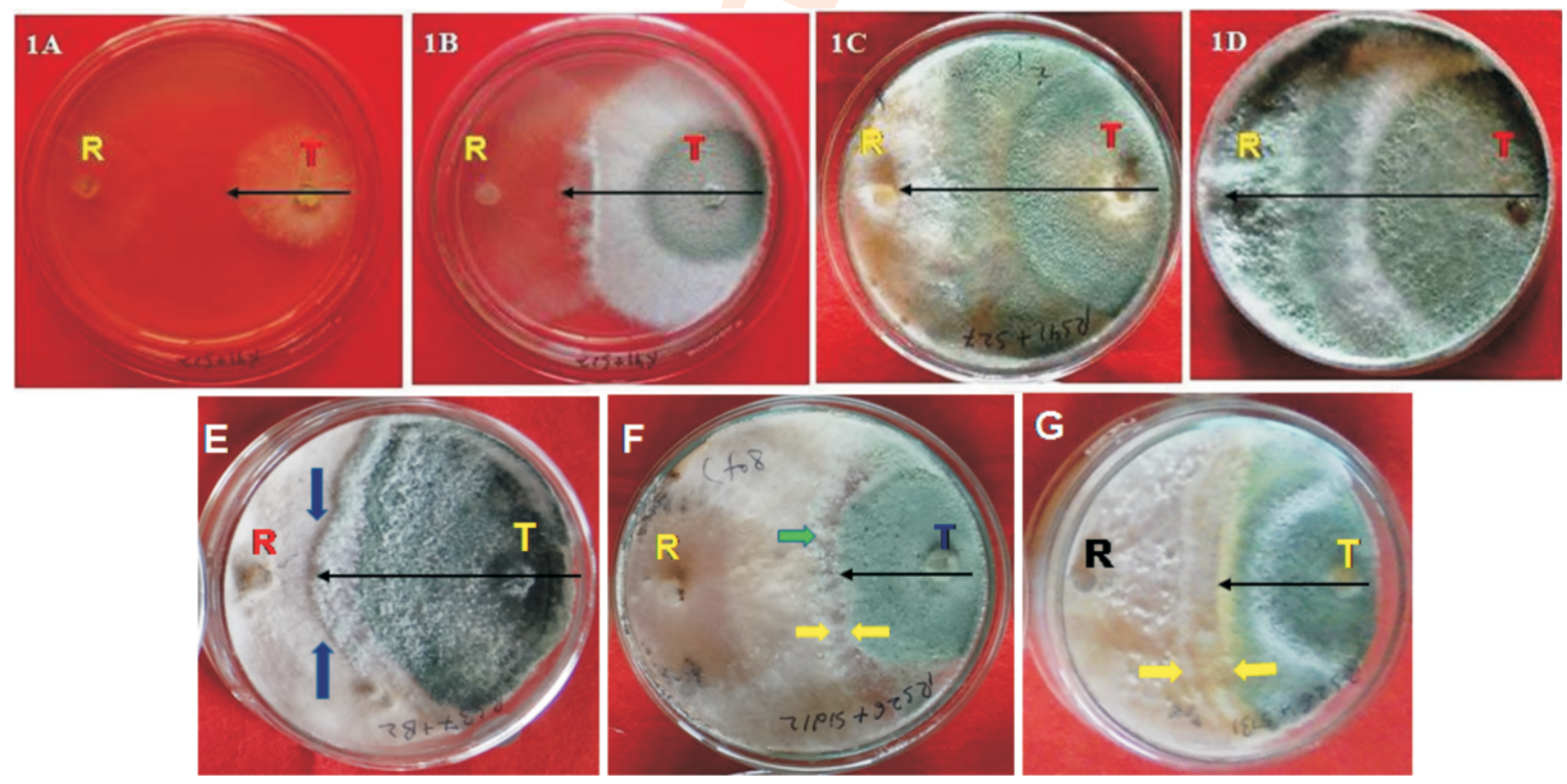

Fig. 1 : Dual plate assay for antagonistic activity of Trichoderma with Rhizoctonia solani: (1A-1D) T. harzianum (SVPRT-THLi06) growth and inhibition of $R$. solani growth after 24, 48, 96 and $120 \mathrm{hr}$, respectively (arrow marks show: Trichoderma growth), (E) T. erinaceum (SVPUAT-TE-1) growth and inhibition of $R$. solani (blue arrow show: Trichoderma overgrowth), (F and G) R. solani growth inhibition by T. harzianum (SVPRT-THL01) and T. atroviride (SVPP-4) respectively (yellow arrow show inhibition zone between them, green arrow show initial sporulation). 


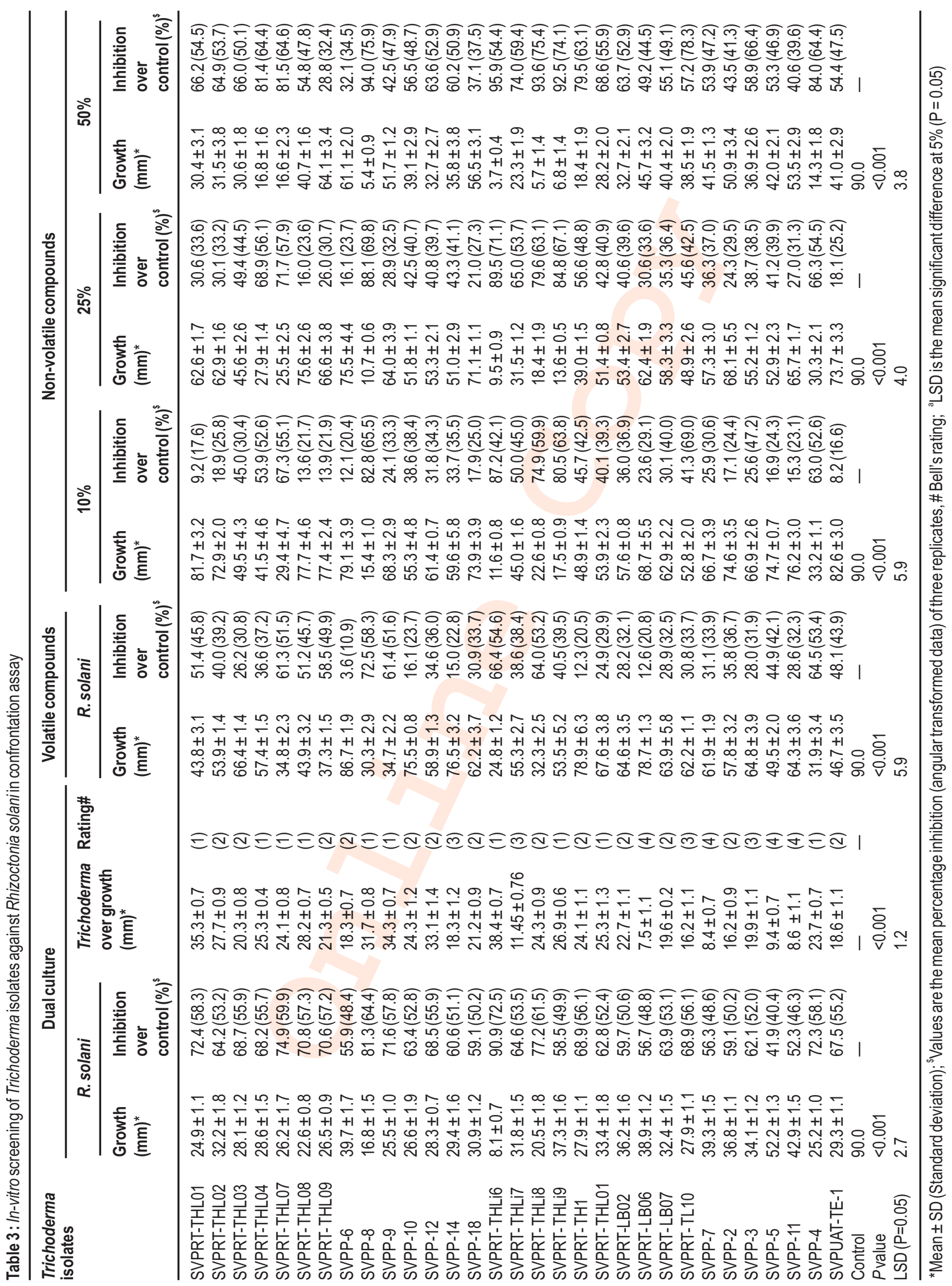


isolate SVPRT- THLi6 showed maximum inhibition at 50\% concentration with an inhibition potential of $95.9 \%$, followed by SVPP-8 with $94.0 \%$ inhibition potential and minimum inhibition potential $(28.8 \%$ ) recorded by SVPRT-THL09 (Table 3). However, even at $10 \%$ concentration, the growth of $R$. solani was significantly inhibited by the culture filtrate compared to the control, suggesting that the SVPRT- THLi6 culture filtrate had a strong inhibitory potential against $R$. solani.
The mycelial growth of $R$. solani was significantly inhibited by volatile compounds produced by $T$. harzianum isolate. T. harzianum isolate (SVPP-8) was effective with highest inhibition rate $(72.5 \%)$, followed by T. harzianum SVPRT-THLi06 and T. atroviride SVPP-4 (66.4\% and 64.5\%), respectively (Table 3). Production of volatile and non-volatile compounds has been associated with the ability of Trichoderma to control phytopathogens (Harman, 2006). Dennis and Webster (1971)
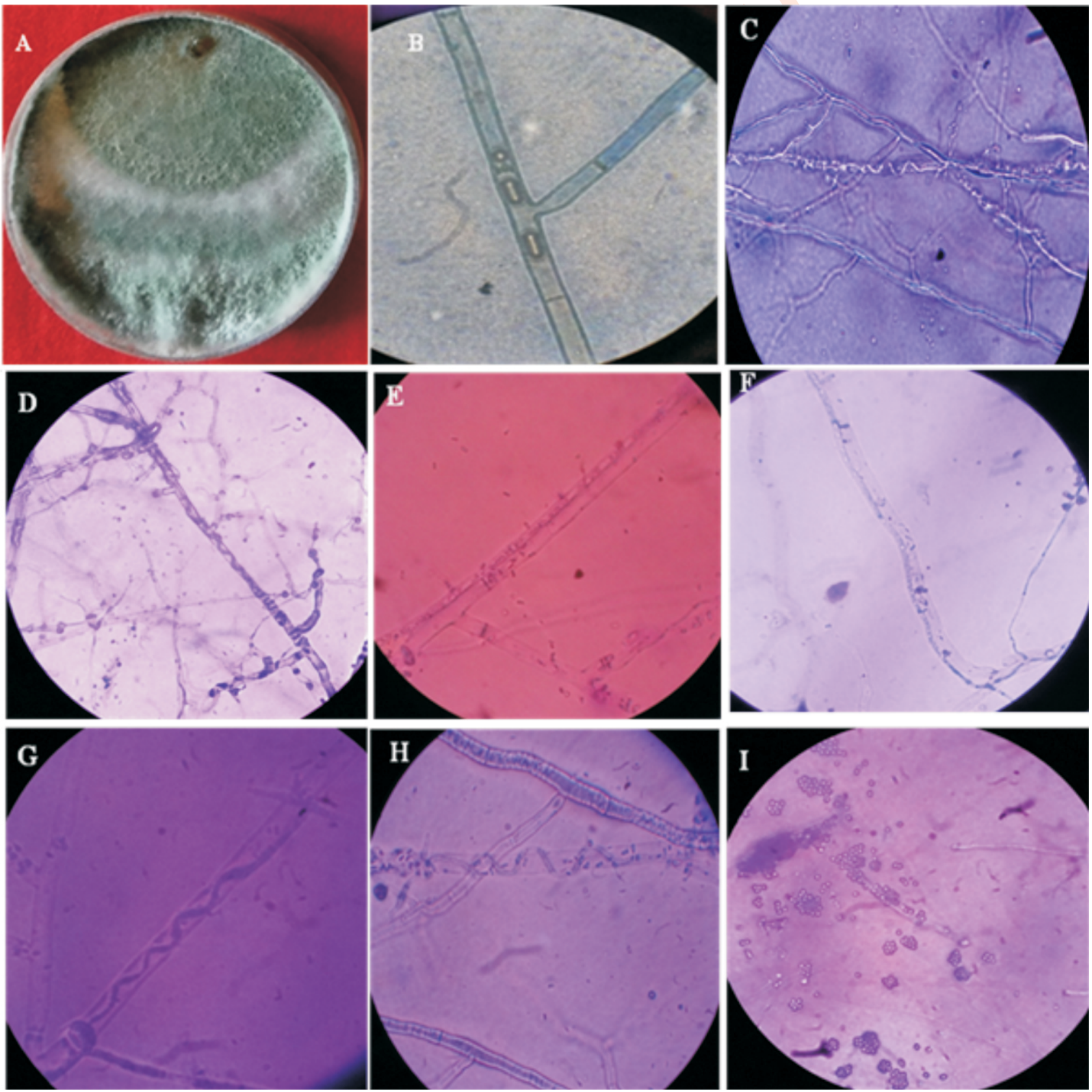

Fig. 2: Photomicrographs showing hyphal interaction between T. harzianum and R. solani; (A) T. harzianum and R. solani interaction in dual culture; (B) Mycelium of $R$. solani; (C, D) T. harzianum hyphae coiling to hyphae of $R$. solani; (E, F) T. harzianum hyphae running parallel to hyphae of $R$. solani; (G, $\mathrm{H}$ ) Lysis of $R$. solani hyphae; (I) Spores of $T$. harzianum attached on hyphae of $R$. solani. 
observed that Trichoderma spp. produce a volatile antibiotic that inhibited mycelial growth of $R$. solani and other test fungi. The volatile and non-volatile metabolites of Trichoderma spp. have also been reported to reduce mycelial growth and sclerotial germination in sclerotial forming fungus by several workers (Srinivasa et al., 2014; Kumar et al., 2017).

The antagonistic potential of five Trichoderma isolates selected by in-vitro screening experiments were further evaluated for their biocontrol of sheath blight disease in-vivo. The selected isolates belonged to three species, i.e., $T$. harzianum, $T$. longibrachiatum and $T$. atroviride. Applications of spore suspension and talk based formulation of $T$. harzianum significantly reduced the disease severity and infected tillers/hills (disease incidence) over control and other Trichoderma spp., irrespective of its mode of application. Significant disease reduction was recorded when all the tested Trichoderma spp. were applied using a mixed mode comprising all three delivery systems together, i.e. soil treatment + seedling root dip + foliar spray followed by dual inoculation of bio-agent using seedling root dip + foliar spray. However, among the T. harzianum treatments, bio-agents SVPRT-THLi06 and SVPP-8 recorded highest value of disease reduction from soil treatment + seedling root dip + foliar spray $(75.1 \%$ and $69.8 \%)$ as compared to fungicide propiconazole 25EC @ 0.1\% which showed disease reduction of $83.9 \%$ (Table 4). Moreover, T. atroviride isolate (SVPRT-TVir01) showed disease reduction over disease severity $(63.0 \%)$, when applied with soil treatment and foliar spray. No significant difference in disease severity was recorded between soil treatment + foliar spray and seedling root dip + foliar spray with all the treatments. However, among different treatments, maximum and significant higher disease severity $(34.5 \%)$ and incidence $(59.4 \%)$ were recorded with soil treatment + foliar spray of the bioagent SVPRT-TL10. Tewari and Singh (2005) reported that maximum rice sheath blight disease control could be achieved when the bioagents were applied through soil, seedling root dip and foliar spray. Furthermore, Verma et al. (2007) reported that Trichoderma spp. can be efficiently used as spores or conidia, which are more lenient to adverse environmental conditions, in contrast to their mycelial and chlamydospore forms as microbial propagules.

The observations of growth parameters revealed that Trichoderma spp. treated rice plants significantly enhanced the rice plant growth performance (Table 4). A significant increase in plant height was observed in all the Trichoderma spp. treatments. The plant heights ranged from $80.4-124.7 \mathrm{~cm}$ while in propiconazole 25 EC (0.1\%) treated plants and control were
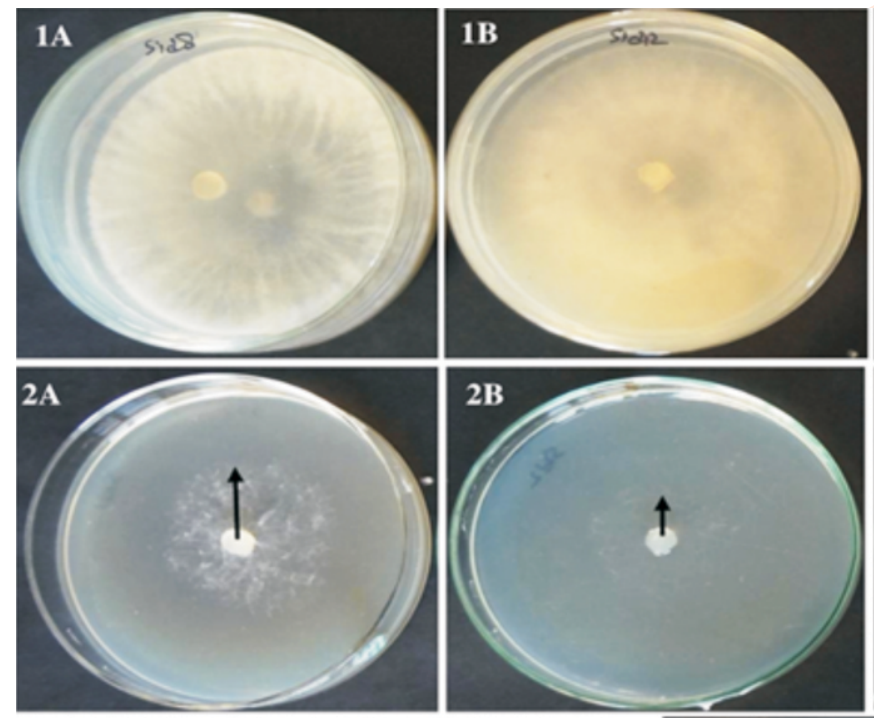
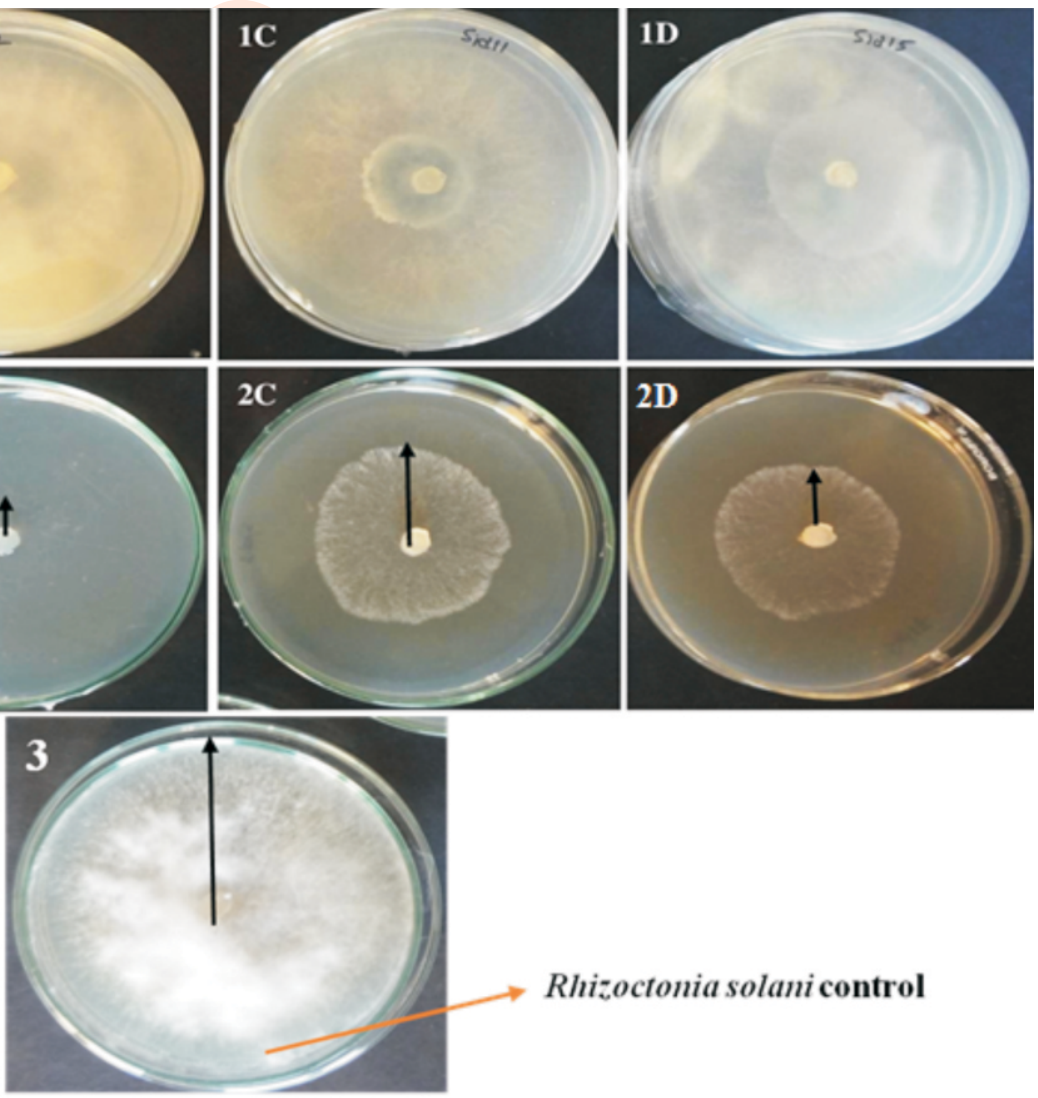

Rhizoctonia solani control

Fig. 3 : In-vitro effect of volatile compounds released by Trichoderma isolates on $R$. solani. 1A-1D: Inoculation of $R$. solani upper side and Trichoderma at lower side. 2A-2D: Growth inhibition of $R$. solani growth after 7DOI. 3: $R$. solani control. Arrow indicate growth of $R$. solani 


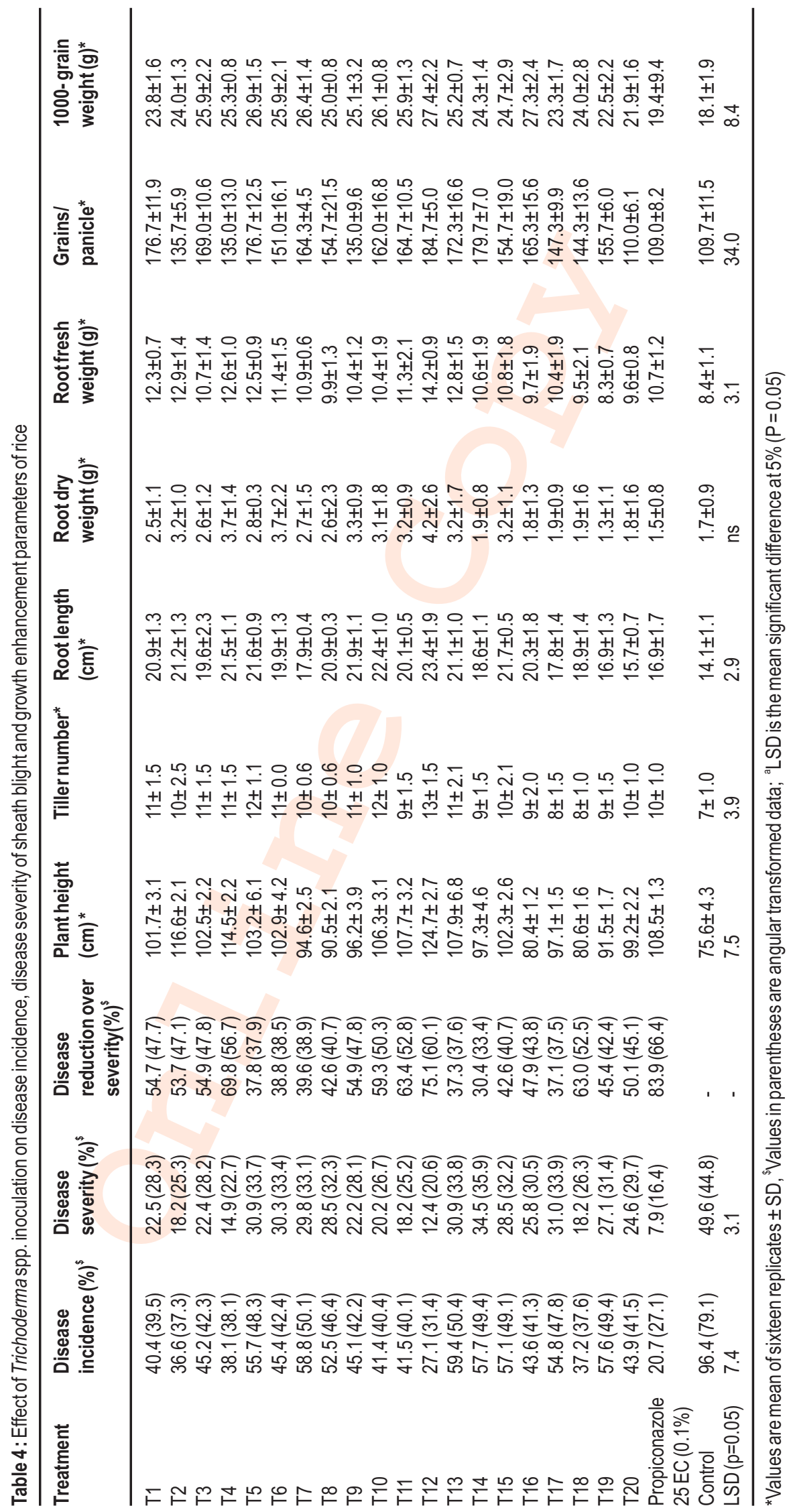

- Journal of Environmental Biology, September 2020 
recorded at $108.5 \mathrm{~cm}$ and $75.6 \mathrm{~cm}$, respectively. However, isolate SVPRT-THLi06 recorded highest increase in plant height (124.7 $\mathrm{cm}$ ) with the treatment soil + seedling root dip + foliar spray followed by isolate SVPRT-THLi04 $(114.5 \mathrm{~cm})$ with soil and foliar spray treatment. Significantly more number of tillers was also recorded for Trichoderma spp. treated rice plants. The highest number of tillers (13) was recorded for isolate SVPP-4 (soil + seedling root dip + foliar spray) treated plants and the lowest number of tillers (8) for SVPRT-TL10 (soil treatment+ foliar spray) treated plants. On the other hand, the propiconazole $25 \mathrm{EC}$ $(0.1 \%)$ treatment and control recorded a tiller number of 10 and 7 , respectively. Root length was found to be significantly increased for SVPRT-THLi06 (soil + seedling root dip + foliar spray) treated plants with $23.4 \mathrm{~cm}$, while the propiconazole $(0.1 \%)$ and control recorded root length of $16.9 \mathrm{~cm}$ and $14.1 \mathrm{~cm}$, respectively. $T$. harzianum isolate SVPRT-THLi06 (soil + seedling root dip + foliar spray) treated rice plants recorded the highest value of root fresh weight $(14.2 \mathrm{~g})$, while for propiconazole $25 \mathrm{EC}(0.1 \%)$ treated plants and control were $10.7 \mathrm{~g}$ and $8.4 \mathrm{~g}$, respectively. Significantly higher no. of grains/panicle was also observed for Trichoderma spp. treated rice plants compared to propiconazole 25 EC $(0.1 \%)$ treated plants and control. Isolate SVPRT-THLi06 (soil + seedling root dip + foliar spray) treated rice plants registered the highest no. of grains/panicle which was 179.7, while for propiconazole 25 EC $(0.1 \%)$ treated plants and control were 109.0 and 109.7, respectively. Significant increase in weight of 1000 grains was observed for all the Trichoderma spp. treatments compared to propiconazole $25 \mathrm{EC}(0.1 \%)$ treated rice plants and control. The highest no. weight of 1000 grains was recorded with SVPRT-THLi06 (soil + seedling root dip + foliar spray) treated rice plants of $27.4 \mathrm{~g}$ while for propiconazole $25 \mathrm{EC}$ $(0.1 \%)$ treated plants and control were $19.4 \mathrm{~g}$ and $18.1 \mathrm{~g}$, respectively. Among the Trichoderma spp. SVPRT-THLi06 (soil + seedling root dip + foliar spray) treated rice plants showed the highest boost in plant height, root length, root fresh weight, no. of grains/panicle and weight of 1000 grains, while Trichoderma spp. SVPP-4 (soil + seedling root dip + foliar spray) had the greatest effect in enhancing the tiller numbers. However, no significant improvement in root dry weight was seen for the Trichoderma spp. treated rice plants.

Rice plants treated with Trichoderma spp. significantly increased rice growth parameters. Higher plant height was observed in Trichoderma spp. inoculated rice plants as compared to control. Saba et al. (2012) suggested that Trichoderma treated rice plants have better nutrient ability that enhance the physiological processes within the rice plants, leading to excellent growth performance. Significantly higher tiller number was recorded in Trichoderma spp. treated rice plants compared to control. Doni et al. (2014) also found that Trichoderma sp. SL2 inoculation could increase tiller number in rice. Furthermore, Shukla et al. (2012) reported that T. harzianum significantly increased rice plant ability to tolerate drought stress and increase water holding capacity, believed to be important factors that led to higher tiller number. IAA produced by $T$. virens and $T$. atroviridae were found to stimulate the growth of lateral roots of Arabidopsis (Contreras-Cornejo et al., 2009). In this study, Trichoderma sp. treated rice plants recorded impressive increase in root length and root fresh weight compared to non-inoculated rice plants. Trichoderma isolates induced fresh and dry root weight of inoculated rice plants. Swain et al. (2018) observed that Trichoderma spp. treated rice plants showed better growth performance as compare to control.

Trichoderma spp. may be used as an eco-friendly and sustainable tool to enhance yield of different crop plants, including rice (Hossain et al., 2017). Application of $T$. harzianum and $T$. viride significantly improved millable canes over control plants (Srivastava et al., 2006). After inoculating with $T$. koningii or $T$. hamatum, crop productivity increase up to $300 \%$ in the field (Sanjeev et al., 2014). Recently, Doni et al. (2018) reported that Trichoderma-based biofertilizer increased rice yield under system of rice intensification (SRI) conditions by $30 \%$ at very low cost ha ${ }^{-1}$. However, the accurate reason for enhanced yields seems to be unclear yet, but in most cases, it is probably due to greater supply of nutrients by Trichoderma spp. to rice plants (Hossain et al., 2017). Yedidia et al. (2001) suggested that presence of plant growth promoting fungi in the rhizosphere increases root surface area allowing the roots to explore larger volume of soil; thus, more nutrients are available to the plants, especially under nutrient-stressed soil environments. Some Trichoderma spp. strains show abilities to improve photosynthetic efficiency (Doni et al., 2014). All of these capabilities singly or in combination contribute to improve crop yield.

It may be concluded that Trichoderma spp. have the potential to biocontrol the sheath blight disease caused by $R$. solani AG1-IA and enhance rice plant growth parameters. Among the T. harzianum strains, SVPRT-THLi06 was the most effective strain having great potential to control sheath blight and rice plant growth promotion compared to other strains tested. Therefore, this strain can be used for production and development of $T$. harzianum based biofertilizers-cum biopesticide to manage Rhizoctonia disease and growth regulator for plants. Furthermore, studies are needed on this promising T. harzianum strain to identify potential metabolites and evaluate possible mode of actions related to biocontrol and plant growth.

\section{Acknowledgments}

The authors are highly grateful to the Department of Recombination Techniques, College of Biotechnology, SVP University of Agriculture and Technology, Meerut for providing laboratory facilities to carry out this investigation. The corresponding author is also grateful to the University Grant Commission, Government of India for financial support during the period of this research. 


\section{References}

Abo-Elyousr, K.A.M., S.I.I. Abdel-Hafez and I.R. Abdel-Rahim: Isolation of Trichoderma and evaluation of their antagonistic potential against Alternaria porri. J. Phytopathol., 162, 1e8 (2014).

Asad, S.A., N. Ali, A. Hameed, S.A. Khan, R. Ahmad, M. Bilal, M. Shahzad and A. Tabassum: Biocontrol efficacy of different isolates of Trichoderma against soil borne pathogen. Polish J. Microbiol., 63, 95-103 (2014).

Bell, D.K., H.D. Wells and C.R. Markham: In-vitro antagonism of Trichoderma species against six fungal pathogens. Phytopathol., 72, 379-382 (1982).

Chaudhary, S., S. Sagar and M. Lal: A comprehensive overview on sheath blight disease of rice and its management. Agricultura, 111$112,145-174(2019)$.

Contreras-Cornejo H.A., L.M. Rodríguez, C.C. Penagos and J.L. Bucio: Trichoderma virens, a plant beneficial fungus, enhances biomass production and promotes lateral root growth through an auxindependent mechanism in Arabidopsis. Plant Physiol., 149, 15791592, (2009).

Dass, A., S. Chandra, A.K. Choudhary, G. Singh and S. Sudhishri: Influence of field re-ponding pattern and plant spacing on rice root and shoot characteristics, yield, and water productivity of two modern cultivars under SRI management in Indian Mollisols. Paddy Water Environ., 14, 45-59 (2016).

Dass, A., K. Shekhawat, A.K. Choudhary, S. Sepat, S.S. Rathore, G. Mahajan and B.S. Chauhan: Weed management in rice using crop-competition. Crop Prot., 95, 45-52 (2017).

Dennis, C. and J. Webster: Antagonistic properties of species-groups of Trichoderma. II. Production of volatile antibiotics. Trans. Brit. Mycol. Soc., 57, 41-48(1971).

Doni, F., A. Isahak, C.R.C.M. Zain and W.M.W. Yusoff: Physiological and growth response of rice plants (Oryza sativa L.) to Trichoderma spp. inoculants. AMB Express, 4, 45 (2014).

Doni, F., C.R. Zain, A. Isahak, F. Fathurrahman, A. Anhar, W.N. Mohamad, W.M. Yusoff and N. Uphoff: A simple, efficient, and farmer friendly Trichoderma-based biofertilizer evaluated with the SRI rice management system. Org. Agricul., 8, 207-223 (2018).

Elad, Y., I. Chet and Y. Henis: Parasitism of Trichoderma spp. on Rhizoctonia solani and Sclerotium rolfsii-scanning electron microscopy. Phytopathol., 73, 85-88 (1983).

Groth, D.E. and J.A. Bond: Effects of cultivars and fungicides on rice sheath blight, yield and quality. Plant Dis., 91, 1647-1650 (2007).

Groth, D.E., M.C. Rus, G.G. Giesler and C.A. Hollier: Foliar fungicides for use in the management of rice diseases. Louisiana Agricultural Experiment Station Bulletin No. 840 (1993).

Hajieghrari, B.: Effect of some Iranian Trichoderma isolates on maize seed germination and seedling vigour. Afr. J. Biotechnol., 9, 43424347 (2010).

Harman, G.E., C.R. Howell, A. Viterbo, I. Chet and M. Lorito: Trichoderma species-opportunistic, avirulent plant symbionts. Nature Rev. Microbiol., 2, 43-56 (2004).

Harman, G.E.: Overview of mechanism and uses of Trichoderma spp. Phytopathol., 96, 190-194 (2006).

Helfish, A.I.A.I., N. Singh, S. Raina and B.D. Singh: Evaluation of Trichoderma isolates against Rhizoctonia solani and Rhizoctonia oryzae causing sheath blight of rice. Plant Dis. Res., 32, 36-46 (2017).

Hossain, M.M., F. Sultana and S. Islam: Plant growth-promoting fungi
(PGPF): Phytostimulation and induced systemic resistance. In: Plant-Microbe Interaction in Argo-Ecological Perspectives (Eds.: D.P. Singh, H.B. Singh and R. Prabha). Springer Nature, Singapore, pp.135-191 (2017).

Inbar, J., J. Menende and I. Chet: Hyphal interaction between Trichoderma harzianum and Sclerotinia sclerotiorum and its role in biological control. Soil Biol. Biochem., 29, 757-763 (1996).

Jariwala, S., V. Kumari and R. Bharat: Antagonistic activity of some fungi against Alternaria solani and Drechlera oryzae. Acta Botanica Indica, 19, 217-223 (1991).

Jeyarajan R., G. Ramakrishnan, D. Dinakaran and R. Sridar: Development of products of Trichoderma viride and Bacillus subtilis for biocontrol of root-rot diseases. In: Biotechnology in India (Ed.: B.K. Dwivedi). Bioved Research Society, Allahabad, pp. 25-36 (1994).

Khan, A.A. and A.P. Sinha: Screening of Trichoderma spp. against Rhizoctonia solani the causal agent of rice sheath blight. Indian Phytopathol., 60, 450-456 (2007).

Kotasthane, A., T. Agrawal, R. Kushwah and O.V. Rahatkar: In-vitro antagonism of Trichoderma spp. against Sclerotium rolfsii and Rhizoctonia solani and their response towards growth of cucumber, bottle gourd and bitter gourd. Eur. J. Plant Pathol., 141, 523-543 (2015).

Kumar, M.K.P., D.K.S. Gowda, R. Moudgal, N.K. Kumar, K.T.P. Gowda and K. Vishwanath: Impact of fungicides on Rice production in India. In: Fungicides-Showcases of Integrated Plant Disease Management from around the World. pp. 7-78 (2013).

Kumar, R., A. Sinha, S. Srivastava and G. Mahajan: Evaluation of biocontrol potential of some fungal decomposers of Sesbania aculeate L. green manure against some soil-borne plant pathogens. J. Environ. Biol., 38, 37-45 (2017).

Laha, G.S., R. Singh, D. Ladhalakshmi, S. Sunder, M.S. Prasad, C.S. Dagar and V.R. Babu: Importance and management of rice diseases: A global perspective. In: Rice Production Worldwide (Eds.: B.S. Chauhan, K. Jabran and G. Mahajan), Springer International Publishing AG, Switzerland, pp. 306-360 (2017).

Mahato, S., S. Bhuju and J. Shrestha: Effect of Trichoderma viride as biofertilizer on growth and yield of wheat. Malaysian J. Sustain. Agricult., 2, 1-5 (2018).

Mahivanan, N., K. Srinivasan and S. Chelliah: Biological control of soilborne diseases of cotton, eggplant, okra and sunflower by Trichoderma viride. J. Plant Dis. Protect., 107, 235-244 (2000).

Mostafa, K.M.D. and A.K.M. Shahjahan: Trichoderma in rice field soils and their effect on Rhizoctonia solani. Bangl. J. Bot., 24, 75-79 (1995).

Prasad, L., S. Chaudhary, S. Sagar and A. Tomar: Mycoparasitic capability of diverse native strain of Trichoderma spp. against Fusarium oxysporum f. sp. Iycopersici. J. Appl. Nat. Sci., 8, 769776 (2016)

Raman, J.: Response of Azotobacter, Pseudomonas and Trichoderma on growth of apple seedling. International Conference on Biological and Life Sciences IPCBEE, IACSIT Press, Singapore. (2012).

Richa, K., I.M. Tiwari, M. Kumari, B.N. Devanna, H. Sonah, A. Kumari, R. Nagar, V. Sharma, J.R Botel and T.R. Sharma: Functional characterization of novel chitinase genes present in the sheath blight resistance QTL: qSBR11-1in Rice Line Tetep. Front. Plant Sci., 7, 1-10 (2016).

Saba, H.D.V., M. Manisha, K.S. Prashant, H. Farham and A. Tauseff: 
Trichoderma - a promising plant growth stimulator and biocontrol agent. Mycosphere, 3, 524-531 (2012).

Sanjeev, K., T. Manibhushan and R. Archana: Trichoderma: Mass production, formulation, quality control, delivery and its scope in commercialization in India for the management of plant diseases. Afr. J. Agricul. Res., 9, 3838-3852 (2014).

Saravanakumar, K., S.V. Arasu and K. Kathiresan: Effect of Trichoderma on soil phosphate solubilization and growth improvement of Avicennia marina. Aquatic Bot., 104, 101-105 (2013).

Shukla, N., R.P. Awasthi, L. Rawat and J. Kumar: Biochemical and physiological responses of rice (Oryza sativa L.) as influenced by Trichoderma harzianum under drought stress. Plant Physiol. Biochem., 54, 78-88 (2012).

Sivakumar, D., W.R.S. Wilson, R.L.C. Wijesundera, F.M.T. Marikar and M. Abeyesekere: Antagonistic effect of Trichoderma harzianum on postharvest pathogens of rambutan (Nephelium lappaceum). Phytoparasit., 28, 1-8 (2000).

Srinivasa, N., T.P. Devi, S. Sudhirkumar, D. Kamil, J.L. Borah and N. Prabhakaran: Bioefficacy of Trichoderma isolates against soilborne pathogens. Afr. J. Microbiol. Res., 8, 2710-2723 (2014).

Srivastava, S., V. Singh, P.S. Gupta, O.K. Sinha and A. Baitha: Nested PCR assay for detection sugarcane grassy shoot phytoplasma in the leafhopper vector Deltocephalus vulgaris: A first report. Plant Pathol., 55, 25-28 (2006).

Sudhakar, R., K.C. Rao and C.S. Reddy: Studies on antagonism of Trichoderma and Gliocdadium specis against $R$. solani of rice. Ind. J. Plant Protec., 26, 25-29 (1998).

Swain, H., T. Adak, A.K. Mukherjee, P.K. Mukherjee, P. Bhattacharyya, S. Behera, T.B. Bagchi, R. Patro, Shasmita, A. Khandual, M.K. Bag,
T.K. Dangar, S. Lenka and M. Jena: Noval Trichoderma strains isolated from tree barks as potential biocontrol agents and biofertilizers for direct seeded rice. Micriobiol. Res., 214, 83-90 (2018).

Taheri, P. and S. Tarighi: A survey on basal resistance and riboflavininduced defence responses of sugar beet against Rhizoctonia solani. J. Plant Physiol., 168, 1114-1122 (2011).

Tewari, L. and R. Singh: Biological control of sheath blight of rice by Trichoderma harzianum using different delivery systems. Indian Phytopathol., 58, 35-40 (2005).

Verma, M., S.K. Brar, R.D. Tyagi, R.Y. Surampalli and J.R. Valero: Antagonistic fungi, Trichoderma spp.: Panoply of biological control. Biochem. Engin. J., 37, 1-20 (2007).

Willocquet, L., M. Noel, H.R. Sackville and S. Savary: Susceptibility of rice to sheath blight: An assessment of the diversity of rice germplasm according to genetic groups and morphological traits. Euphytica, 183, 227-241 (2012).

Yadav, R.N., D. Mishra, N.W. Zaidi, U.S. Singh and H.B. Singh: Biocontrol efficacy of Trichoderma spp. against the major diseases of rice (Oryzae sativa L.). Int. J. Agricul. Environ. Biotech., 11, 543548 (2018).

Yadav, S., G. Anuradha, R.R. Kumar, L.R. Vemireddy, R. Sudhakar, K. Donempudi, D. Venkata, F. Jabeen, Y.K. Narasimhan, B. Marathi and E.A. Siddiq: Identification of QTLs and possible candidate genes conferring sheath blight resistance in rice (Oryza sativa L.). SpringerPlus, 4, p. 175 (2015).

Yedidia, I., A.K. Srivastva, Y. Kapulnik and I. Chet: Effect of Trichoderma harzianum on microelement concentrations and increased growth of cucumber plants. Plant Soil, 235, 235-242 (2001). 